\title{
O uso de objetos educacionais digitais: A transposição didática tecnológica digital dos produtos educacionais da educação profissional e tecnológica
}

\author{
The use of digital educational objects: The digital technological didactic transposition of \\ educational products in professional and technological \\ El uso de objetos educativos digitales: La transposición didáctica tecnológica digital de productos \\ educativos en la educación profesional y tecnológica
}

Recebido: 10/01/2022 | Revisado: 14/01/2022 | Aceito: 17/01/2022 | Publicado: 17/01/2022

Fernanda Alves Costa

ORCID: https://orcid.org/0000-0002-0889-725X Instituto Federal de Educação, Ciência e Tecnologia do Tocantins, Brasil E-mail: fernandaalves@ifto.edu.br

Weimar Silva Castilho

ORCID: https://orcid.org/0000-0002-5642-6049 Instituto Federal de Educação, Ciência e Tecnologia do Tocantins, Brasil E-mail: weimar@ifto.edu.br

Mary Lúcia Gomes Silveira de Senna ORCID: https://orcid.org/0000-0002-4112-5470 Instituto Federal de Educação, Ciência e Tecnologia do Tocantins, Brasil E-mail: marysenna@ifto.edu.br

Rivadavia Porto Cavalcante

ORCID: https://orcid.org/0000-0002-6568-7910 Instituto Federal de Educação, Ciência e Tecnologia do Tocantins, Brasil E-mail: riva@ifto.edu.br

Rosa Maria Machado de Sena

ORCID: https://orcid.org/0000-0002-3616-650X Instituto Federal de Educação, Ciência e Tecnologia do Tocantins, Brasil

E-mail: rosa.sena@ifto.edu.br

\begin{abstract}
Resumo
Este artigo aborda o uso dos objetos educacionais digitais como campo de inter-relação entre a educação e as tecnologias digitais, tornando o saber científico em objeto do saber a ser ensinado. $\mathrm{O}$ texto aponta a importância dos produtos educacionais produzidos na perspectiva da Educação profissional e Tecnológica para a formação ampla e integral do estudante no ensino médio. Para tanto buscou se por meio de pesquisa bibliográfica a conceituação e o entendimento sobre os objetos educacionais digitais e a teoria da transposição didática tecnológica digital na educação. Realizou-se também uma análise de dados através dos resultados obtidos pela revisão bibliográfica das publicações científicas (dissertações/produtos educacionais) publicadas na página web do Mestrado em Educação Profissional e Tecnológica do IFTO - Campus Palmas. Suscitou-se ainda uma breve discussão sobre o uso das tecnologias digitais na educação como possível recurso para a formação ampla e integral dos jovens na atualidade. Em sua conclusão o artigo revela que os diversos objetos educacionais digitais produzidos possibilitam caminhos norteadores no processo de ensino e aprendizagem.
\end{abstract}

Palavras-chave: Educação; Tecnologia; Transposição didática; Produtos educacionais; Ensino.

\begin{abstract}
This article addresses the use of digital educational objects as a field of interrelation between education and digital technologies, turning scientific knowledge into an object of knowledge to be taught. The text points out the importance of educational products produced in the perspective of Vocational and Technological Education for the broad and integral formation of the student in high school. In order to do so, the conceptualization and understanding of digital educational objects and the theory of digital technological didactic transposition in education were sought through bibliographic research. A data analysis was also carried out through the results obtained by the bibliographic review of scientific publications (dissertations/educational products) published on the web page of the Masters in Professional and Technological Education of the IFTO - Campus Palmas. A brief discussion was also raised on the use of digital technologies in education as a possible resource for the broad and comprehensive training of young people today. In its conclusion, the article reveals that the various digital educational objects produced enable guiding paths in the teaching and learning process.
\end{abstract}

Keywords: Education; Technology; Didactic transposition; Educational products; Teaching. 


\begin{abstract}
Resumen
Este artículo aborda el uso de los objetos educativos digitales como campo de interrelación entre la educación y las tecnologías digitales, convirtiendo el conocimiento científico en un objeto de conocimiento para ser enseñado. El texto señala la importancia de los productos educativos producidos en la perspectiva de la Educación Profesional y Tecnológica para la formación amplia e integral del estudiante de secundaria. Para ello se buscó a través de la investigación bibliográfica la conceptualización y comprensión de los objetos educativos digitales y la teoría de la transposición didáctica tecnológica digital en la educación. También se realizó un análisis de datos a través de los resultados obtenidos por la revisión bibliográfica de publicaciones científicas (disertaciones/productos educativos) publicadas en la página web de la Maestría en Educación Profesional y Tecnológica de la IFTO - Campus Palmas. También se planteó una breve discusión sobre el uso de las tecnologías digitales en la educación como posible recurso para la formación amplia e integral de los jóvenes de hoy. En su conclusión, el artículo revela que los diversos objetos educativos digitales producidos posibilitan orientar caminos en el proceso de enseñanza y aprendizaje.
\end{abstract}

Palabras clave: Educación; Tecnología; Transposición didáctica; Productos educativos; Enseñanza.

\title{
1. Introdução
}

As instituições de educação básica enfrentam o grande desafio da transfiguração do processo de ensino devido a imersão dos jovens em um mundo tecnológico digital, cada vez mais fluido e inovador, obtendo novas habilidades cognitivas, aprendendo fora dos intramuros escolares, sendo assim, a busca pelos professores na incorporação e transmissão dos conteúdos via internet, parte do princípio que qualquer conhecimento tem que ter validade dentro dos saberes científicos para posteriormente serem elaborados os objetos educacionais digitais transformando-se no saber ensinado. (Yves Chevallard, 1991, Da Rosa \& Locateli, 2018, 1991; Rocha \& Lima, 2021)

Quando falamos do uso das tecnologias educacionais é necessário considerar as ações públicas já realizadas neste sentido e quais foram os obstáculos enfrentados nesse processo, utilizados como exemplos, os produtos educacionais da Educação profissional e Tecnológica, são elaborados na perspectiva da formação integral humana, em suas diversas dimensões, cognitiva, estética, afetiva, didática, cultural, estética e do mundo do trabalho através da cultura, educação e tecnologia, voltados para serem instrumentos utilizados pelas instituições, professores e estudantes da educação básica. Partindo do trabalho científico (dissertação) consolidado e aprovado pela comunidade científica que atendendo a transposição didática dos saberes que alcança o estudante, tornando este protagonista do processo de aprendizagem. (Demerval Saviani 1989; Gaudêncio Frigotto et al., 2005; Da Rosa \& Locateli, 2018)

A diferença entre os mestrados profissionais e os mestrados acadêmicos como explica Ostermann \& Rezende (2009) é que o primeiro visa a qualificação para o mercado de trabalho, tendo como objetivo formar profissionais que no futuro saibam utilizar a pesquisa de modo a agregar valor a suas atividades e de seus semelhantes, já o segundo destinam-se apenas na formação voltada para a pesquisa.

Partindo desta exposição, este trabalho levanta os seguintes problemas: Quais produtos educacionais são objetos educacionais digitais? Quais os elementos teóricos e metodológicos contidos nos produtos educacionais necessários para a transposição didática tecnológica digital? Quais produtos educacionais são voltados para tecnologia assistiva digital?

Sendo o ProfEPT, um programa de pós-graduação em Educação Profissional e Tecnológica com um curso de mestrado profissional em Educação Profissional e Tecnológica ofertado em rede nacional, pertencente à área de Ensino e reconhecido pela Coordenação de Aperfeiçoamento de Pessoal de Nível Superior - CAPES do Ministério da Educação com objetivo de proporcionar formação em educação profissional e tecnológica, visando tanto a produção de conhecimentos com o desenvolvimento de produtos, por meio da realização de pesquisas que integrem os saberes inerentes ao mundo do trabalho e ao conhecimento sistematizado.

Esta pesquisa justifica-se pela necessidade da divulgação para os professores e profissionais das instituições de ensino básico sobre os produtos educacionais da educação profissional e tecnológica, especificamente sobre a utilização como objetos educacionais digitais, sendo ferramentas de caráter complementar e suplementar nos ensinamentos de disciplinas e na interação 
dos trabalhos multidisciplinares e enfatizando sua importância na formação integral dos cidadãos através da transposição didática tecnológica digital.

Este artigo, tem como objetivo de elaborar uma base de conhecimentos sobre os produtos educacionais e as dissertações publicadas e aprovadas pelo Mestrado em Educação Profissional e Tecnológica (ProfEPT) do IFTO - Campus Palmas, como também, apontar os produtos educacionais que podem ser utilizados como recursos educacionais digitais e discutir os aspectos das teorias de aprendizagem e metodologias que auxiliam no processo de ensino e aprendizagem.

\section{Metodologia}

A pesquisa é imprescindível na formalização e sistematização para produção das diversas etapas do desenvolvimento científico, sendo seu objetivo a descoberta de respostas para problemas por meio de procedimentos científicos, a pesquisa social é o processo que utiliza a metodologia científica para obtenção de novos conhecimentos no campo da realidade social. (Antônio Carlos Gil, 2008; Uwe Flick et al., 2009)

A pesquisa exploratória é necessária para coleta de dados desta pesquisa, assim, este trabalho tem como finalidade esclarecer e discutir sobre o uso dos objetos educacionais digitais (OEDs) na produção dos produtos educacionais do Mestrado Profissional em Educação Profissional e Tecnológica (ProfEPT).

Tendo em vista a formulação de hipóteses para estudos posteriores sobre as dissertações publicadas até o mês de novembro de 2021 pelo IFTO - Campus Palmas (Instituição associada ao ProfEPT) a partir da integração do uso de ferramentas da tecnologia da informação e comunicação no uso educacional em perspectiva da transposição didática tecnológica digital voltada para professores e estudantes da educação básica. (Antônio Carlos Gil, 2008; Eva Maria Lakatos \& De, 2003)

A abordagem qualitativa é recomendada para interpretar fenômenos realísticos, vivenciais e sociais através da interação entre a observação e a formulação do conceito, entre o desenvolvimento teórico e a pesquisa e entre a explicação e a percepção. A pesquisa exploratória é uma metodologia que costuma envolver o levantamento bibliográfico com as experiências com o problema pesquisado através de análise de exemplos que estimulem a compreensão. (Bulmer, 2001; Gibbs, 2008)

Sendo assim, este estudo tem como perspectiva a pesquisa exploratória, de abordagem qualitativa que utiliza procedimentos de revisão bibliográfica. A pesquisa bibliográfica voltada sobre entendimento sobre a transposição didática tecnológica digital, através da identificação dos produtos educacionais que são OEDs.

Quanto à análise de dados será através dos resultados obtidos pela revisão bibliográfica através das publicações científicas (dissertações) publicadas na página web do Mestrado em Educação Profissional e Tecnológica do IFTO - Campus Palmas, a forma de apresentação dos resultados será feita por meio da elaboração de análise crítica através das discussões dos dados levantados.

\section{Resultados e Discussão}

Os resultados deste estudo foram obtidos por informações e por publicações de trabalhos científicos que podem ser acessados pela página desenvolvida para instituição associada do Estado do Tocantins (http://www.ifto.edu.br/profept), de forma clara e objetiva onde são encontradas as informações e atividades desenvolvidas.

As dissertações e os produtos educacionais dissecados nesta pesquisa possuem algumas características em comum: o uso de tecnologias digitais na preparação e execução dos procedimentos de pesquisa, a realização de atividades de investigação por meio de experimentação prática com estudantes do ensino médio integrado, a construção de sequências didáticas voltada para professores através de uma indagação central voltada para desenvolvimento de atividades práticas voltadas para ciências 
aplicadas e sociais, contendo aspectos de conteúdo, pedagógicos e tecnológicos, e, por fim, a formação do estudante sob a perspectiva da formação humana integral, trabalhando suas diversas dimensões através da cultura, educação e tecnologia. (Demerval Saviani 1989; Gaudêncio Frigotto et al., 2005; Charlot, 2014; Barbosa et al., 2016)

Os resultados das dissertações da pesquisa bibliográfica analisadas contendo produtos educacionais que são objetos educacionais digitais são apresentados no Quadro 1.

Quadro 1. Produtos educacionais como objetos educacionais digitais.

\begin{tabular}{|c|c|c|c|}
\hline AUTOR & DISSERTAÇÃO & PRODUTO EDUCACIONAL & TIPO DE OED \\
\hline Correia (2021) & $\begin{array}{l}\text { O ensino de polinômios com o } \\
\text { software GeoGebra }\end{array}$ & $\begin{array}{c}\text { Caderno de atividades - O ensino de } \\
\text { polinômios com o software } \\
\text { GeoGebra }\end{array}$ & $\begin{array}{c}\text { E- book } \\
\text { (Hipertexto/Site) }\end{array}$ \\
\hline D. Santos (2021) & $\begin{array}{l}\text { O uso da WebQuest na análise de } \\
\text { erros matemáticos }\end{array}$ & $\begin{array}{l}\text { WEBQUEST: Decifrando o } \\
\text { Coronavírus através da Matemática }\end{array}$ & $\begin{array}{c}\text { WebQuest } \\
\text { (Hipertexto/Site) }\end{array}$ \\
\hline G. Santos (2021) & $\begin{array}{l}\text { Letramentos para construção da } \\
\text { autonomia do Técnico de Nível } \\
\text { Médio em Agropecuária: uma } \\
\text { proposta Didática para o Ensino } \\
\text { Híbrido da comunicação oral- } \\
\text { argumentativa }\end{array}$ & $\begin{array}{l}\text { Proposta Didática para o ensino } \\
\text { híbrido da comunicação oral- } \\
\text { argumentativa no ensino médio } \\
\text { integrado }\end{array}$ & Vídeo \\
\hline Santos Junior (2021) & $\begin{array}{l}\text { Pegadas da inclusão: um jogo de } \\
\text { tabuleiro digital em Libras para o } \\
\text { contexto do Ensino Médio Integrado }\end{array}$ & $\begin{array}{l}\text { Pegadas da Inclusão: um Jogo de } \\
\text { Tabuleiro Digital em Libras (PDI) }\end{array}$ & Jogo digital \\
\hline Pontes (2021) & $\begin{array}{c}\text { Uso da Robótica Educacional como } \\
\text { suporte ao Ensino e Aprendizagem de } \\
\text { Lógica de Programação }\end{array}$ & $\begin{array}{l}\text { Robótica educacional: uma } \\
\text { abordagem prática no ensino de } \\
\text { lógica de programação }\end{array}$ & $\begin{array}{c}\text { E- book } \\
\text { (Hipertexto/Site) }\end{array}$ \\
\hline Maia (2021) & $\begin{array}{l}\text { Utilização dos jogos didáticos para o } \\
\text { Ensino Aprendizagem de conceitos } \\
\text { estatísticos em uma turma de Ensino } \\
\text { Médio Integrado }\end{array}$ & Jogos estatísticos & Jogo digital \\
\hline Aguiar (2021) & $\begin{array}{c}\text { A inserção e a formação omnilateral e } \\
\text { integral do estudante surdo nas aulas } \\
\text { de Física }\end{array}$ & Libras em 1 minuto e meio & Vídeo \\
\hline Castro (2020) & $\begin{array}{c}\text { Sala de aula invertida: O uso da vídeo } \\
\text { aula no ensino de sociologia }\end{array}$ & $\begin{array}{l}\text { SALA DE AULA INVERTIDA: } \\
\text { Roteiro didático: o uso da vídeo } \\
\text { aula no ensino de sociologia }\end{array}$ & Vídeo \\
\hline Oliveira (2020) & $\begin{array}{l}\text { A WEBQUEST como ferramenta } \\
\text { tecnológica da aprendizagem baseada } \\
\text { em problemas: proposta de aplicação } \\
\text { ao estudo do biogás no curso técnico } \\
\text { em meio ambiente }\end{array}$ & "WebBiogasQuest" & $\begin{array}{c}\text { Webquest } \\
\text { (Hipertexto/Site) }\end{array}$ \\
\hline
\end{tabular}




\begin{tabular}{|c|c|c|c|}
\hline Silva (2020) & $\begin{array}{c}\text { APLICATIVO FinCalc: uma } \\
\text { Estratégia Educativa para Aprender } \\
\text { Matemática Financeira }\end{array}$ & Aplicativo FinCalc & Aplicativo \\
\hline Guimarães (2020) & $\begin{array}{c}\text { A prática educomunicativa na } \\
\text { formação integral: a produção de } \\
\text { podcast no IFTO Campus Araguatins }\end{array}$ & Áudio \\
\hline
\end{tabular}

Fonte: Autores (2021).

O objeto educacional digital (OED) pertencem a uma classe especial dos objetos de aprendizagem (learning objects), sendo qualquer recurso digital destinado ao processo de ensino e aprendizagem, geralmente, armazenados em repositórios online, sendo populares os exemplos de OED como: simulações, animações, hipertextos, aplicativos, áudios, games e vídeos. (Portella, 2019)

Os OED podem ser utilizados como ferramentas de caráter complementar e suplementar as aulas de qualquer disciplina, sendo necessária para sua implementação o uso de estratégias pedagógicas e metodológicas adequadas, quanto à sua utilização não pode ser pensada como algo capaz de resolver todos os problemas encontrados no ensino, possuindo potencialidade quando está relacionado com base no planejamento didático favorável em determinadas circunstâncias. (Portella, 2019)

O uso dos dispositivos de acesso à internet e as ferramentas da tecnologia de informação e comunicação são instrumentos ricos e possibilitam a incorporação na educação através de recursos pedagógicos que viabilizem e maximizam o conhecimento. (Souza \& Barbosa, 2013).

Os produtos educacionais apresentados são disponibilizados no repositórios digitais abertos do Portal eduCapes (https://educapes.capes.gov.br/handle/1884/34734), permitindo a sua localização por meio de sites de buscas, a utilização de abordagem construcionista destes como objetos educacionais digitais tem como perspectiva a abertura para que o estudante experimente, simule, brinque, construa conhecimentos e habilidades a partir de um ambiente compatível com tais ações e no contexto preparado para tal. (Nobre, 2011)

Nas dissertações disponibilizadas observa-se a preocupação que a utilização das tecnologias digitais pelos professores preconize o desenvolvimento de metodologias educativas que oferecem mais oportunidade de aprendizagem e cooperação com os estudantes. (Nobre, 2011)

As produções audiovisuais utilizadas como ferramentas no processo de ensino e aprendizagem são apontadas como os OEDs mais utilizados nos trabalhos, sendo a plataforma Youtube onde ocorre a maior disponibilidade de vídeos como recurso didático, com uma grande variedade de vídeos educativos em diversas áreas, como também, com diversos formatos como: vídeos-aulas, vídeos demonstrativos, históricos, informativos, divertidos entre outros. Sendo que sua utilização esteja de acordo com os critérios didáticos e necessidades voltados para os estudantes, sendo exigido do professor realize busca criteriosa por estes conteúdos, como também a questão de vídeos curtos que promovam a proximidade com os estudantes, na tentativa de evitar a dispersão destes. (Portella, 2019).

\subsection{Transposição didática tecnológica digital}

Segundo o regulamento do Mestrado Profissional em Educação Profissional e Tecnológica em Rede Nacional (ProfEPT) visa a produção de conhecimento através do desenvolvimento de produtos educacionais com a realização de pesquisas que integram os saberes inerentes ao mundo do trabalho e ao conhecimento sistematizado.

Propondo alcançar os seguintes objetivos: atendimento de formação continuada em uma perspectiva interdisciplinar 
voltada para os profissionais da Rede Federal de Educação Profissional e Tecnológica no desenvolvimento de atividades de ensino, gestão e pesquisa em vista à inovação tecnológica, formação em nível nacional de recursos humanos na perspectiva de contribuir com o desenvolvimento socioeconômico, científico e cultural nas diversas regiões do Brasil e o desenvolvimento de trabalhos de investigação interdisciplinar, constituído pela interface entre Trabalho, Ciência, Cultura e Tecnologia, na perspectiva de melhoria dos processos educativos e de gestão em espaços formais ou não-formais.

A CAPES (Brasil, 2013), descreve como devem ser os produtos educacionais, caracterizando-os como: "uma sequência didática, um aplicativo computacional, um jogo, um vídeo, um conjunto de vídeo-aulas, um equipamento, uma exposição etc.".

Sendo analisado por Moreira e Nardi o produto educacional é (p.04, 2009): "algo identificável e independente da dissertação". Os autores ressaltam que apesar das dissertações serem frutos dos trabalhos de pesquisa sobre os produtos, porém estes têm sua 'própria identidade' devendo ser disponibilizados na página do programa, para que possam ser analisados e utilizados pelos diversos profissionais da área educacional, oportunizando melhorias na educação básica.

Sendo assim, Rosa e Locatelli entendem que (p. 27, 2018): “os produtos educacionais representam o elo entre os conteúdos escolares e sua efetivação no processo de aprendizagem”. As autoras enfatizam que o processo de adaptação se assemelha a proposta de 'transposição didática' do estudioso Yves Chevallard.

Para um objeto do saber (savoir savant) ser considerado pelo sistema de ensino é necessário que sua inserção seja útil para o sistema didático, ocorrendo essa inserção, o saber científico torna-se agora em objeto do saber a ser ensinado (savoir à ensigner), que finalmente transforma-se em saber ensinado (savoir ensigné), no sentido de mudar de circunstância. A necessidade de ensinar o conhecimento científico de forma a ser absorvido pelo estudante exige do professor estratégias que transformam o conhecimento em algo que possa ser ensinado. (Yves Chevallard, 1991)

No processo de adaptação dos conteúdos voltados para as atividades de ensino, não é necessário apenas simplificá-los para eliminar o difícil ou o abstrato, para o ensino desses conteúdos nas instituições escolares, sendo exigido transformá-los em algo que possa ser ensinado com conceitos, experiências e linguagens próprias. Enfatizado por Pinho Alves (2000) que o saber científico foi desenvolvido e publicado ao longo de muitos anos por muitos personagens, passou pela crítica, reformulações, aceitações e legitimação de outros cientistas.

O aumento do uso das ferramentas da tecnologia da informação e comunicação e a inserção de novas modalidades de ensino como o ensino remoto ou ensino híbrido provocaram mudanças significativas na educação básica brasileira, fazendo com que professores e estudantes lidem com os processos de ensino e aprendizagem via internet. Sendo assim, as buscas por sequências didáticas, ferramentas, objetos educacionais digitais e metodologias para auxiliar e colaborar para maior participação e motivação dos estudantes. (Rocha \& Lima, 2021)

Conforme Sousa e Menezes (2019) temos que refletir e discutir acerca das mudanças e contribuições do uso tecnologias digitais nas atividades de ensino, das metodologias empregadas e funcionamento das práticas pedagógicas, a inserção destas tecnologias não garantem o sucesso ou na adaptação do estudante sendo necessária a incorporação e integração no planejamento e no currículo escolar. Sendo assim, requer-se mudanças na transposição didática envolvendo todos os autores no processo de ensino e aprendizagem, provocando as instituições de ensino no sentido de 'entender' os novos contextos educacionais vivenciados pelos estudantes.

As tecnologias digitais na educação possibilitam a melhoria e agregação de valores aos processos que afetam a qualidade do ensino, a questão é como podemos aproveitá-las para melhorar os processos de ensino e aprendizagem, um primeiro passo é de fato procurar entender essas tecnologias, participação dos professores através de capacitações incentivadas pelos gestores escolares, ou seja, demonstrar que uso em sala de aula destas tecnologias podem auxiliar estudantes no entendimento de conteúdo. 
Conforme Yves Chevallard (1991) favorecendo a Transposição Didática Externa (TDE), relacionada com as transformações que ocorrem na 'escola fora da escola' e a com a Transposição Didática Interna (TDI), que ocorre nos ambientes escolares (espaços formais e não-formais).

Partindo do contexto do uso das tecnologias digitais na educação é bastante difundido e discutido o uso das metodologias ativas que segundo Moran (2018, p. 4): "São estratégias de ensino centradas na participação efetiva dos estudantes na construção da aprendizagem, de forma flexível, interligada e híbrida”. Ressaltado também pelo autor que essas metodologias em um mundo conectado e digital se expressa por meio de modelos híbridos com muitas possíveis combinações.

Sendo assim, os produtos educacionais no formato digital são ferramentas de aproximação entre conteúdos desenvolvidos para serem objetos de ensino e o atendimento das demandas de aprendizagem dos estudantes, sendo estes gerados para atender as demandas das diferentes dimensões de natureza curricular, cognitiva, afetiva, didática, cultural, estética, do mundo do trabalho, dentre outras. (Da Rosa \& Locateli, 2018)

Contribuindo na concretização dos processos de ensino e aprendizagem buscando alcançar o sistema educacional através de instrumentos que possam atender, auxiliar e alcançar professores e estudantes em suas diversas tarefas, sendo que essas concepções teóricas indo encontro ao campo epistemológico didático, sociológico até o psicológico, com suas teorias de aprendizagem. (Da Rosa \& Locateli, pg. 30, 2018)

\subsection{Produtos educacionais voltados para educação inclusiva}

Os resultados das dissertações da pesquisa bibliográfica analisadas contendo produtos educacionais que são objetos educacionais digitais tendo como perspectiva a tecnologia assistiva são apresentados no Quadro 2.

Quadro 2. Produtos educacionais voltados para tecnologia assistiva digital.

\begin{tabular}{|c|c|c|}
\hline Autor & Produto educacional & OED \\
\hline Santos Junior (2021) & $\begin{array}{c}\text { Pegadas da Inclusão: um Jogo de } \\
\text { Tabuleiro Digital em Libras (PDI) }\end{array}$ & Vídeo \\
\hline Aguiar (2021) & Libras em 1 minuto e meio & Jogol \\
\hline
\end{tabular}

Fonte: Autores (2021).

A segregação social, as desigualdades, as vulnerabilidades e a marginalização preconceituosa com as pessoas deficientes têm raízes históricas que envolve a discussão sobre o reconhecimento da sua condição humana, política, de participação ativa sob as necessidades de liberdade, interação e expressão, os aspectos históricos da tecnologia assistiva (TA) vem de uma longa experiência de luta social através do reconhecimento por processo ontológico, onde uma totalidade reconstrói a partir da diferença, reconhecido que a origem da TA deve-se por fins militares, onde a mesma vinha no sentindo reabilitar pessoas que foram vítimas na segunda guerra mundial, a inclusão prevista na (TA) busca garantir os direitos de liberdade, dignidade, comunicação, mobilidade, acessibilidade e igualdade social (condição política e cidadã), sendo marcada pela luta contra a política de exclusão. (Conte et al., 2017)

A tecnologia assistiva (TA) é considerada como área de conhecimento, com características transdisciplinares, onde produtos, recursos, metodologias, estratégias, práticas e serviços que tem como objetivo a promoção da funcionalidade, relacionada às atividades e participação das pessoas com deficiências, com incapacidades ou mobilidade reduzida, na busca 
por autonomia, independência, qualidade de vida e inclusão social. (BRASIL, 2009)

A política da educação inclusiva provocou o progresso de pesquisas voltadas para criação e desenvolvimento de recursos e métodos educacionais com a perspectiva de atender as especificidades dos estudantes desde à educação básica até o ensino superior, com intuito de superação de barreiras da comunicação, melhoria da funcionalidade e mobilidade, buscando a autonomia pessoal, dos movimentos sensoriais e mentais pela procura da inclusão social plena. (Conte et al., 2017)

$\mathrm{O}$ atendimento da diversidade educacional têm que considerar as limitações cognitivas e motoras dos estudantes, oportunizando a compreensão e o reconhecimento da própria condição humana: em seus interesses, sensibilidades, motivações, singularidades, olhares e experiências, sem abandono ou sem retirar as diferenças deste mundo, porém, promovendo diálogos culturais de liberdade, pluralidade e espontaneidade na formação do reconhecimento e formação social, como também derrubar mitos quanto ao uso da tecnologias educacionais no processo de aprendizagem e possibilitar o acesso à tecnologia assistiva. (Conte et al., 2017)

O uso de objetos educacionais digitais na educação inclusiva possibilita que ferramentas pedagógicas sejam acessíveis no processo de aprendizagem dos estudantes, sendo estes protagonistas deste processo, pois não basta saber escolher, é preciso a contribuição das práticas de ensino para êxito do uso destas ferramentas. (Souza \& Barbosa, 2013)

\section{Conclusão}

O processo da transposição didática tecnológica digital tem como premissa a possibilidade de integração de softwares nos processos de ensino e aprendizagem, partindo do conhecimento científico ao saber a ser ensinado, esta nova configuração no ambiente escolar estimula o desenvolvimento de projetos de ensinos relacionados com a criação, produção e divulgação de objetos educacionais digitais.

Os produtos educacionais da Educação Profissional e Tecnológica tornam-se exemplo e referência sobre o uso das tecnologias digitais no ambiente escolar da educação básica brasileira, possuindo estudos pautados na formação humana integral dos estudantes, trazendo modelos que podem ser aplicados por professores e instituições em uma perspectiva interdisciplinar.

Diante do reconhecimento da integração tecnológica digital na educação, a possibilidade de sucesso apresentado pelos produtos educacionais, sugere-se o desenvolvimento de projetos e ações na educação básica voltado ao acesso aos dispositivos conectados via internet (computadores, tablets, celulares, etc.) e de forma concomitante a capacitação para uso dos recursos tecnológicos digitais por professores, estudantes e os diversos profissionais das instituições da educação pública.

\section{Agradecimentos}

Ao Instituto Federal de Educação, Ciência e Tecnologia do Tocantins (IFTO) pelo apoio prestado durante o desenvolvimento das etapas deste trabalho.

\section{Referências}

Aguiar, E. (2021). A inserção e a Formação Omnilateral e integral do Estudante Surdo nas Aulas de Física (W. Castilho, Ed.). http://www.ifto.edu.br/profept/dissertacoes/dissertacao-evaneide-de-brito-feitosa-aguiar-1.pdf/view

Antônio Carlos Gil. (2008). Métodos e técnicas de pesquisa social. São Paulo Atlas.

Barbosa, M. V., Miller, S., \& Mello, S. A. (2016). Teoria histórico-cultural: questões fundamentais para a educação escolar. Faculdade de Filosofia e Ciências. https://doi.org/10.36311/2016.978-85-7983-772-2

Brasil. (2013). Documento de área 2013. CAPES. http://www.avaliacaotrienal2013.capes.gov.br/documento-de-area-e-comissao.

Brasil. (2009). Secretaria Especial dos Direitos Humanos. http://www.pessoacomdeficiencia.gov.br/app/sites/default/files/publicacoes/livro-tecnologiaassistiva.pdf" 
Bulmer, M. (2001). Sociological research methods : an introduction. Macmillan.

Castro, I. (2020). Sala de aula invertida: O uso da videoaula no ensino de sociologia (W. Castilho, Ed.). http://www.ifto.edu.br/profept/dissertacoes/dissertacao-ibanes-castro.pdf/view

Charlot, B. (2014). Trabalho e educação: abordagens antropológica e sócio-histórica da relação com o saber às práticas. Ed. Cortez.

Conte, E., Ourique, M. L. H., \& Basegio, A. C. (2017). Tecnologia assistiva, direitos humanos e educação inclusiva: uma nova sensibilidade. Educação Em Revista, 33(0). https://doi.org/10.1590/0102-4698163600

Correia, D. (2021). O ensino de polinômios com o software GeoGebra (W. Souza, Ed.). http://www.ifto.edu.br/profept/dissertacoes/dissertacao-divanez-alvescorreia.pdf/view

Rosa, C. C. T. W., \& Locateli, A. (2018). Produtos Educacionais: Diálogo Entre Universidade e Escola. Revista ENCITEC, 8(2), 26. https://doi.org/10.31512/encitec.v8i2.2716

Dermeval Saviani. (1989). Sobre a concepção de politecnia [apresentado do "Seminário Choque Teórico" da Saúde, 2, 3 e 4 de dezembro de 1987]. Rio De Janeiro Politécnico Da Saúde Joaquim Venâncio

Gaudêncio Frigotto, Ciavatta, M., Ramos, M., Eliza Bartolozzi Ferreira, Regina, S., \& CorrêaV. (2005). Ensino médio integrado: concepção e contradições. Cortez Editora; Rio De Janeiro, RJ

Gibbs, G. (2008). Análise de Dados Qualitativos. Porto Alegre, Artmed.

Guimarães, K. (2020). A prática educomunicativa na formação integral: a produção de podcast no IFTO Campus Araguatins. http://www.ifto.edu.br/profept/dissertacoes/dissertacao-kelline-guimaraes.pdf/view

Lakatos, E. M. \& Marconi, M. A. (2003). Fundamentos de metodologia científica. Ed. Atlas.

Maia, I. (2021). Utilização dos jogos didáticos para o Ensino Aprendizagem de conceitos estatísticos em uma turma de Ensino Médio Integrado. http://www.ifto.edu.br/profept/dissertacoes/dissertacao-islani-silva-maia.pdf/view

Moran, M. (2018). Metodologia ativas para uma aprendizagem mais profunda. https://curitiba.ifpr.edu.br/wp-content/uploads/2020/08/Metodologias-Ativaspara-uma-Educacao-Inovadora-Bacich-e-Moran.pdf

Moreira, M. A., \& Nardi, R. (2010). O mestrado profissional na área de Ensino de Ciências e Matemática: alguns esclarecimentos. Revista Brasileira de Ensino de Ciência E Tecnologia, 2(3). https://doi.org/10.3895/s1982-873x2009000300001

Nobre, I. (2011). um caminho de possibilidades e desafios. https://educimat.ifes.edu.br/images/stories/Publica\%C3\%A7\%C3\%B5es/Livros/Livro-PIECaminhos-de-Possibilidades-2011.pdf

Oliveira, I. (2020). A WEBQUEST como ferramenta tecnológica da aprendizagem baseada em problemas: proposta de aplicação ao estudo do biogás no curso técnico em meio ambiente. http://www.ifto.edu.br/profept/dissertacoes/dissertacao-ines-garcao.pdf/view

Ostermann, F., \& Rezende, F. (2009). Projetos de desenvolvimento e de pesquisa na área de ensino de ciências e matemática: Uma reflexão sobre mestrados profissionais. Caderno Brasileiro de Ensino de Física, 26(1). https://doi.org/10.5007/2175-7941.2009v26n1p66

Pinho, A. F., Jose de. (2000). Atividades experimentais: do método à prática construtivista/. Repositório Institucional Da UFSC. http://repositorio.ufsc.br/xmlui/handle/123456789/79015

Pontes, P. (2021). Uso da Robótica Educacional como suporte ao Ensino e Aprendizagem de Lógica de Programação. http://www.ifto.edu.br/profept/dissertacoes/dissertacao-paulo-ricardo-da-silva-pontes.pdf/view

Portella, B. (2019). Uma proposta de objeto educacional digital para o ensino de cinemática.

Rocha, E. M., \& Lima, J. M. da S. (2021). Impactos e desafios do ensino on-line decorrentes da pandemia COVID-19. Revista Ibero-Americana De Estudos Em Educação, 16(2), 377-390. https://doi.org/10.21723/riaee.v16i2.14526

Santos Junior, E. (2021). Pegadas da inclusão: um jogo de tabuleiro digital em Libras para o contexto do Ensino Médio Integrado. http://www.ifto.edu.br/profept/dissertacoes/dissertacao-edivaldo-dos-santos-junior.pdf/view

Santos, D. (2021). O uso da WebQuest na análise de erros matemáticos (A. Filho, Ed.). http://www.ifto.edu.br/profept/dissertacoes/dissertacao-dinelise-sousasantos.pdf/view

Santos, G. (2021). Letramentos para construção da autonomia do Técnico de Nível Médio em Agropecuária: uma proposta Didática para o Ensino Híbrido da comunicação oral-argumentativa (R. Cavalcante, Ed.). http://www.ifto.edu.br/profept/dissertacoes/dissertacao-gleyde-ohana-ribeiro-dos-santos.pdf/view

Silva, J. (2020). APLICATIVO FinCalc: uma Estratégia Educativa para Aprender Matemática Financeira. http://www.ifto.edu.br/profept/dissertacoes/dissertacao-josenilma-silva.pdf/view

Souza, R., \& Barbosa, J. (2013). Educação inclusiva, Tecnologia e Tecnologia Assistiva. https://editoracriacao.com.br/wpcontent/uploads/2015/11/inclusiva.pdf

Uwe Flick, Joice Elias Costa, \& Sônia Elisa Caregnato. (2009). Uma introdução à pesquisa qualitativa. Porto Alegre Bookman.

Yves Chevallard. (1991). La transposition didactique : du savoir savant au savoir enseigné. La Pensée Sauvage. 